\title{
O efeito da sazonalidade da precipitação na florística e na estrutura da regeneração natural dos campos rupestres da Serra Norte de Carajás, Pará, Brasil \\ The effect of seasonality of rainfall in the floristic and structure of natural regeneration of the rupestrian fields of the Sierra Norte de Carajás, Pará, Brazil
}

\author{
Priscilla Prestes Chaves' ${ }^{1}$ Leandro Valle Ferreiral \\ IMuseu Paraense Emílio Goeldi/MCTI. Belém, Pará Brasil
}

\begin{abstract}
Resumo: Poucos estudos foram realizados na Amazônia comparando a variação da estrutura e da florística da regeneração natural de plantas em relação à sazonalidade da precipitação, e nenhum foi realizado nos campos rupestres da Amazônia. $\bigcirc$ objetivo deste estudo é comparar a florística e a estrutura da comunidade da regeneração natural dos campos rupestres e das lagoas sazonais, entre os períodos de estiagem e chuvoso, na Serra Norte de Carajás, no estado do Pará. As amostragens ocorreram em dois períodos, chuvoso e estiagem, usando parcelas de $1 \times 1 \mathrm{~m}$, onde todos os indivíduos foram contados e identificados. Nos campos rupestres, foram identificadas 67 espécies, 46 no período de estiagem e 33 no chuvoso. Nas lagoas sazonais, foram identificadas 61 espécies, variando de 30 a 41, entre os períodos de estiagem e chuvoso, respectivamente. Não houve diferença significativa no número de indivíduos e na riqueza e diversidade de espécies nos campos rupestres e nas lagoas sazonais entre ambos os períodos. Contudo, há grande mudança na composição de espécies, nos dois tipos de vegetações, entre os períodos. Isto demonstra que as comunidades da regeneração natural estão claramente adaptadas às variações abióticas provocadas pela variação na quantidade de chuvas.
\end{abstract}

Palavras-chave: Sazonalidade. Regeneração natural. Campos rupestres

Abstract: Few studies have been conducted on Amazon compared to the variation in the structure and floristic communities of natural regeneration relative seasonality of precipitation and no study has been conducted in the rocky fields of the Amazon. The aim of this study is to compare the floristic and structure of natural regeneration community vegetations of rocky fields and seasonal ponds, between periods of drought and rainy in the Sierra Norte de Carajás, Brazil. Sampling occurred in two seasons (rainy and dry). In stony fields were identified 67 species, 46 and 33 species, between dry and rainy seasons, respectively. In Seasonal ponds were identified 61 species, ranging from 30 to 41 species, among the dry and rainy season, respectively. There was no significant difference in the number of individuals and the richness and diversity of species in the rocky fields and seasonal ponds between periods of drought and rainy. However, there is a great change in species composition, in both types of vegetation, between periods of drought and rainy. This demonstrates that communities of natural regeneration in both types of vegetation are clearly adapted to abiotic variations caused by the wide variation in the amount of rainfall.

Keywords: Seasonal. Natural regeneration. Rupestrian fields.

CHAVES, P. P. \& L. V. FERREIRA, 2016. O efeito da sazonalidade da precipitação na florística e estrutura da regeneração natural dos campos rupestres da Serra Norte de Carajás, Pará, Brasil. Boletim do Museu Paraense Emílio Goeldi. Ciências Naturais 11(1): $103-116$.

Autor para correspondência: Leandro Valle Ferreira. Museu Paraense Emílio Goeldi/MCTI. Coordenação de Botânica. Av. Perimetral, 1901 - Terra Firme. Belém, PA, Brasil. CEP 66077-530 (lvferreira@museu-goeldi.br).

Recebido em 06/04/2015

Aprovado em 21/03/2016

Responsabilidade editorial: Maria de Lourdes Pinheiro Ruivo

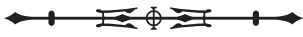




\section{INTRODUÇÃO}

Entre os tipos de vegetação do bioma Amazônia, os campos rupestres são um dos menos estudados. Veloso et al. (1991) definem os campos rupestres como vegetações com estrutura campestre, descontínua e associadas a afloramentos rochosos nas serras do Brasil central e oriental.

Os campos rupestres representam importante ambiente de enclave dentro do domínio da floresta tropical amazônica, levando Veloso et al. (1991) a criarem o termo "refúgios montanos" ou "relíquias de vegetação" para esses enclaves, por se tratarem de vegetações isoladas, em um contexto completamente distinto da flora dominante na região onde se localizam (Vasconcelos, 2011). São vegetações típicas de ambientes montanos, com estrutura arbustiva e/ou herbácea, com comunidades próprias e caracterizadas pelo grande número de espécies endêmicas, como Perama carajensis J.H. Kirkbr., e ainda de espécies ameaçadas de extinção, como Ipomoea cavalcantei D.F. Austin (Convolvulaceae) e Ipomoea carajasensis D.F. Austin. (Convolvulaceae) (Rayol, 2006).

Na Amazônia brasileira, esse tipo de vegetação ocupa somente uma pequena área, sendo representado por enclaves de vegetação com distribuição disjunta, formada por algumas serras de diferentes tamanhos e circundada por vales recobertos por floresta ombrófila densa ou aberta (Secco \& Lobo, 1988; Silva, 1991; Silva et al., 1996).

Os campos rupestres localizados em afloramentos rochosos de óxidos de ferro, denominados de canga couraçada ou canga hematítica, estão localizados na região da Serra dos Carajás, no estado do Pará, entre 500 a 750 metros de altitude. Esses campos apresentam diferentes fisionomias, variando desde áreas abertas recobertas por plantas herbáceas a áreas recobertas, com adensamento de arbustos e pequenas árvores (Silva et al., 1996; Vasconcelos, 2011).

Além do substrato predominante de óxido de ferro, outros tipos de solos estão presentes, tais como plintossolos e cambissolos, associados aos campos rupestres, capões de floresta e organossolos associados às lagoas temporárias (Schaefer et al., 2008).

Estudos botânicos realizados na Serra dos Carajás mostram que os campos rupestres e as lagoas sazonais são caracterizados pelo baixo número de espécies. Contudo, muitas são endêmicas a esses tipos de vegetações (Silva \& Rosa, 1990). Uma das explicações para esse padrão é a alta concentração de metais, principalmente de óxidos de ferro e hematita, que determinam grande pobreza de nutrientes e baixa capacidade de retenção de água nos solos (Silva, 1989), resultando em uma comunidade de plantas com extrema tolerância a esses fatores, o que explica a ocorrência de espécies endêmicas (Silva \& Rosa, 1990; Silva et al., 1996).

Os campos rupestres da Serra dos Carajás estão sobre solos com alto teor de hematita, sendo, portanto, usados na indústria mineral. Os maiores enclaves desse tipo de vegetação estão situados dentro da Floresta Nacional de Carajás, no sudeste do estado do Pará, onde a companhia brasileira mineradora Vale (antiga Vale do Rio Doce) exerce atividades de mineração ligadas à extração de minério de ferro nos campos rupestres.

Por serem um tipo de vegetação de pouca representatividade na Amazônia, com grande quantidade de espécies endêmicas e ameaçadas de extinção, e serem explorados economicamente pela indústria de minérios, os campos rupestres merecem uma proteção especial, pois qualquer perda de habitats pode ter consequências graves para a conservação da biota desse tipo de vegetação.

$\mathrm{Na}$ estratégia para a conservação dos tipos de vegetações dos campos rupestres da Serra dos Carajás é necessário conhecer os mecanismos físicos responsáveis pela regulação da florística e da estrutura da comunidade de plantas e animais, entre os quais a variação da quantidade de precipitação anual pode ser um fator-chave.

Bastos (1996) relata que variação na precipitação é um dos fatores mais importantes para a regulação da comunidade de plantas herbáceas nas vegetações de restingas costeiras na Amazônia paraense. 
Nos campos rupestres de Carajás, a sazonalidade da precipitação é muito nítida. No período chuvoso, as médias de precipitação ultrapassam os $220 \mathrm{~mm}$ mensais, enquanto, no período de estiagem, as médias de precipitação se reduzem drasticamente, ficando abaixo dos $40 \mathrm{~mm}$ mensais (IBAMA, 2003).

Alguns autores relatam que a regeneração natural de comunidade de plantas em vegetações abertas, com alta sazonalidade na precipitação, é um dos principais fatores que regulam a dinâmica da vegetação (Tuomisto \& Ruokolainen, 1994; Tuomisto, 2003).

Bastos (1996) determinou que a composição de espécies da comunidade de plantas herbáceas nas restingas costeiras responde diretamente à sazonalidade da precipitação, resultando em comunidades com espécies exclusivas entre os períodos de estiagem e chuvoso.

Silva (1991), trabalhando nos campos rupestres da Serra dos Carajás, relata que a comunidade de espécies da regeneração natural parece ser adaptada à alta sazonalidade da precipitação ao longo do ano, e que parece haver uma divisão da comunidade de plantas em relação aos períodos de baixa e de alta precipitação mensal.

Esses resultados são fundamentais, a fim de subsidiar outros estudos com a finalidade de traçar estratégias para a conservação da comunidade da regeneração natural. O objetivo do presente estudo é comparar a florística e a estrutura da comunidade da regeneração natural nas vegetações dos campos rupestres e lagoas sazonais entre o período de estiagem e o chuvoso na Serra Norte de Carajás, no estado do Pará, como subsídio à conservação da biota nesse tipo único de vegetação.

\section{MATERIAL E MÉTODOS}

\section{ÁREA DE ESTUDO}

A Floresta Nacional de Carajás está localizada no sudeste

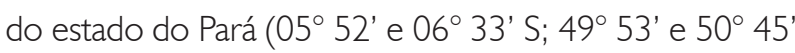
W) e tem 395 mil hectares. Essa unidade faz parte de um mosaico de áreas protegidas, que totalizam cerca de 2 milhões de hectares (Figura 1).

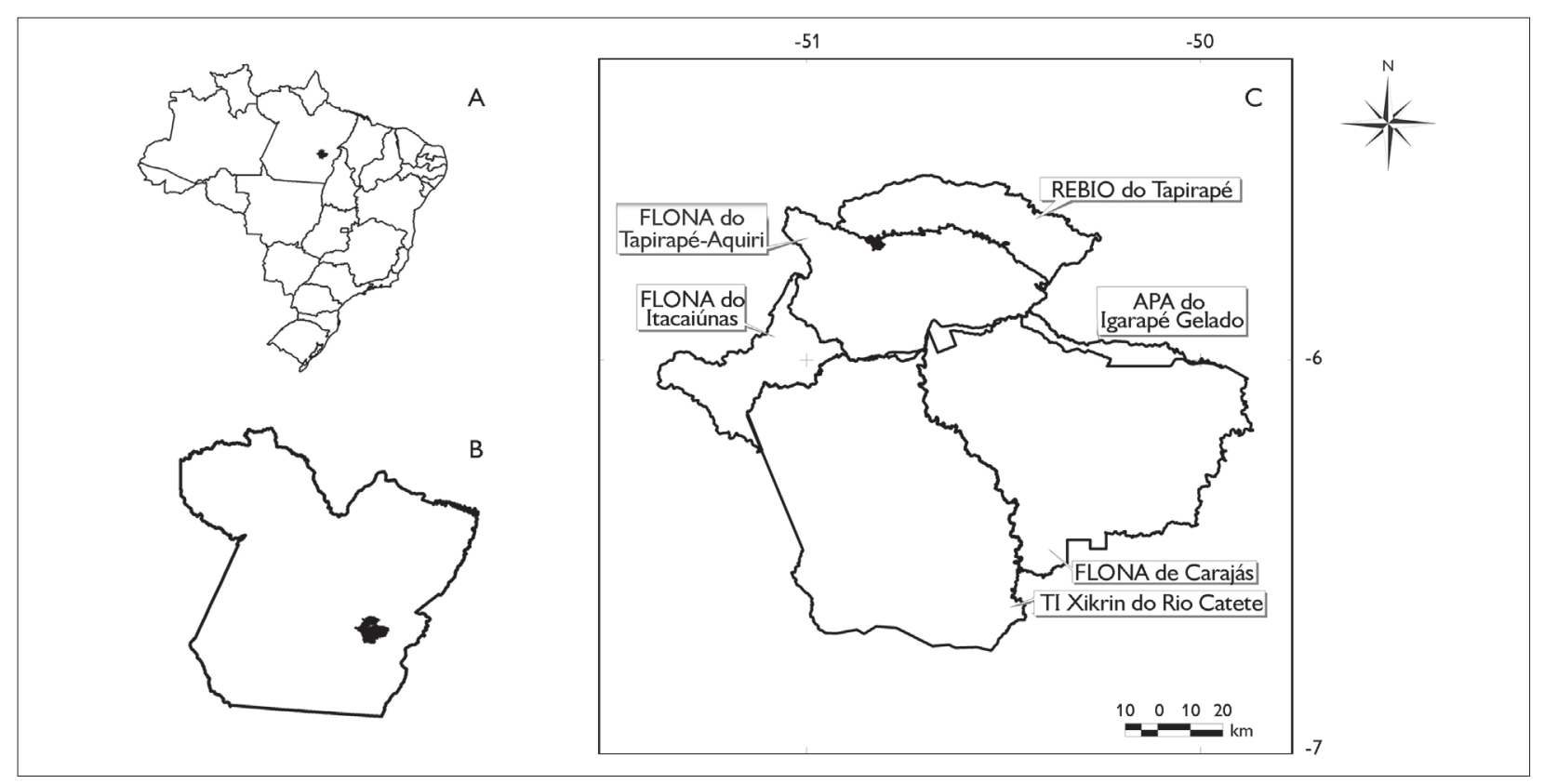

Figura 1. Mapa do Brasil mostrando o estado do Pará (A); localização do mosaico de áreas protegidas em relação ao estado do Pará (B); e tipos de áreas protegidas do mosaico (C). Legendas: REBIO = Reserva Biológica; APA = Área de Proteção Ambiental; FLONA = Floresta Nacional; TI = Terra Indígena.

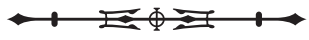


A maior parte da Floresta Nacional de Carajás é representada por floresta ombrófila, dividida nas fisionomias densa, aberta ou aluvial. Contudo, existe um tipo de vegetação denominado de campo rupestre, que cresce sobre substrato de minério de ferro, sendo localmente conhecido como savana metalófica ou vegetação de canga (Secco \& Mesquita, 1983; Silva et al., 1996).

Os campos rupestres da Floresta Nacional de Carajás têm uma distribuição disjunta (não contínua). Esses campos rupestres são representados por pequenos maciços de serras, entre os vales do Xingu e do Araguaia, com estrutura geológica complexa e altitudes que variam de 500 a 800 metros (Ab'Saber, 1986).

O clima na Floresta Nacional de Carajás é dividido em dois grupos: (1) equatorial continental e (2) equatorial mesotérmico de altitude. $\bigcirc$ primeiro ocorre nas áreas de colinas baixas e encostas, enquanto o segundo está associado às serras.
As precipitações anuais variam de 2.000 a 2.400 mm e o tipo climático dos topos das serras é caracterizado por apresentar temperaturas médias de 23 a $25^{\circ} \mathrm{C}$, baixa insolação (4,5 a 5 horas) e ventos moderados.

A precipitação na Floresta Nacional de Carajás é caracterizada pela grande sazonalidade, sendo o período de estiagem (período menos chuvoso) compreendido entre os meses de junho a setembro, com médias de precipitações abaixo dos $40 \mathrm{~mm}$, e o período chuvoso compreendido entre os meses de outubro a maio, com médias de precipitações acima dos $220 \mathrm{~mm}$.

\section{COLETA DE DADOS}

As coletas de dados da comunidade da regeneração natural ocorreram nos meses de junho e agosto de 2009, no período de estiagem, e janeiro e março de 2010, no período chuvoso, em três enclaves de campos rupestres da Serra Norte de Carajás, denominados de N4, N5 e N6 (Figura 2).

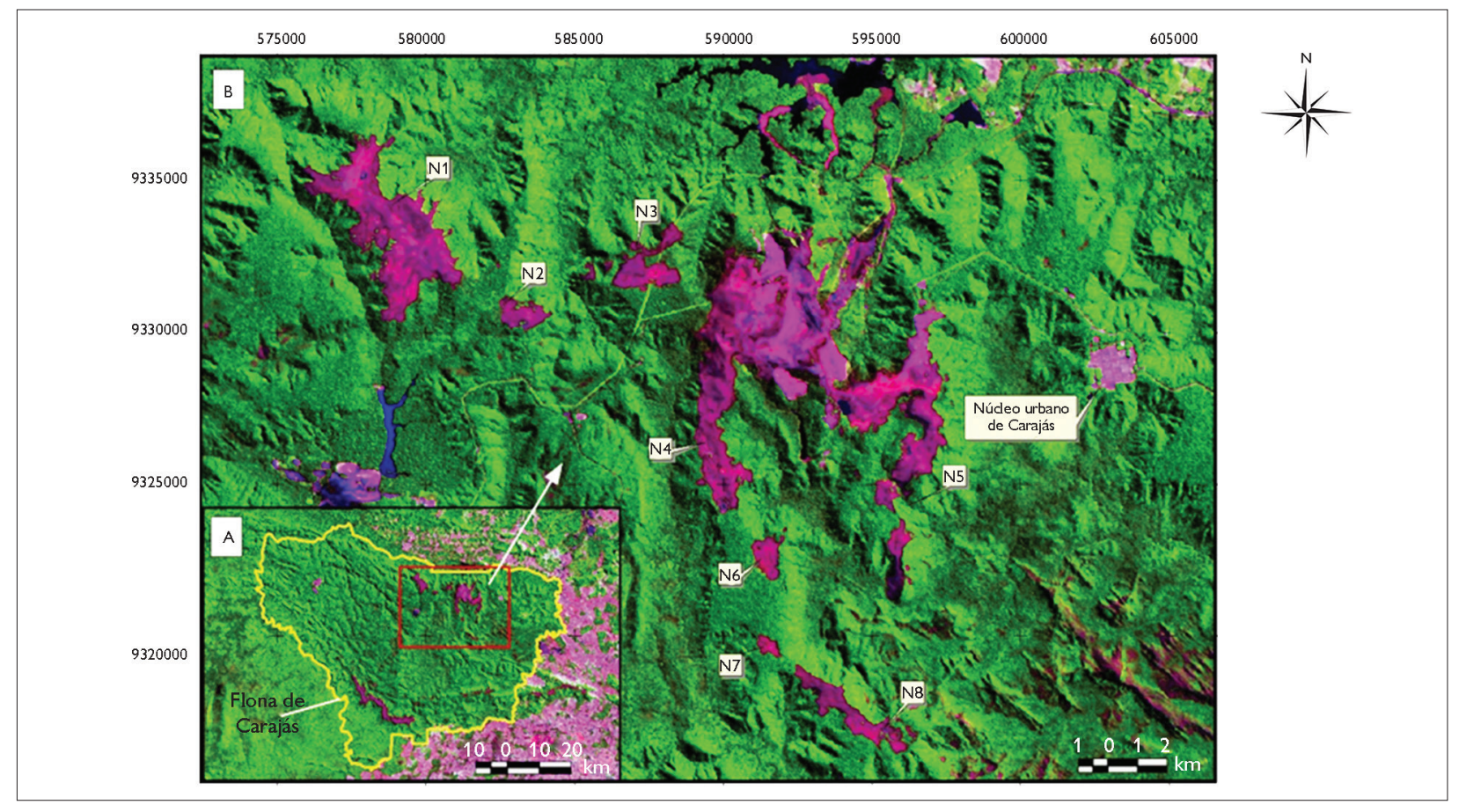

Figura 2. Imagem de satélite mostrando os limites da Floresta Nacional de Carajás (A) e os limites dos campos rupestres (B). Fonte: Museu Paraense Emílio Goeldi.

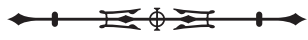


Em cada enclave, foram amostrados dois tipos de vegetações: (1) campos rupestres sobre substrato de minério de ferro e (2) lagoas sazonais.

No levantamento dos campos rupestres, foram usadas 200 parcelas de $1 \times 1$ metro, 100 parcelas no período de estiagem e 100 no período chuvoso. A distribuição das parcelas foi aleatória, com uma distância de 20 metros entre cada.

No levantamento da comunidade das lagoas sazonais, foram usadas 200 parcelas de $1 \times 1$ metro, 100 no período de estiagem e 100 no período chuvoso, distribuídas aleatoriamente nas margens e no centro das lagoas. Em cada parcela, todos os indivíduos foram contados, registrados e identificados. Foi considerado um só espécime para os indivíduos que se organizavam em touceiras.

Todo material botânico fértil coletado está sendo incorporado ao acervo do Herbário João Murça Pires, do Museu Paraense Emílio Goeldi (MG). O sistema de classificação botânica utilizado é APG III (The Angiosperm Phylogeny Group, 2009).

\section{ANÁLISE DE DADOS}

As diferenças da riqueza e diversidade de espécies e densidade de indivíduos (variáveis dependentes) entre os períodos de estiagem e chuvoso (fatores) foram testadas com teste $\mathrm{t}$ de Student. A densidade de indivíduos foi transformada em Log, pois os dados não tinham distribuição normal (Zar, 2010).

\section{RESULTADOS}

\section{CAMPOS RUPESTRES}

Foram identificadas 67 espécies no período de estiagem, e 46 e 33 no chuvoso (Apêndice 1). Não houve variação significativa da riqueza de espécies entre o período de estiagem $(X=4,5$; $D P=1,55)$ e chuvoso $(X=4,1$; $D P$ $=1,64)(\mathrm{t}=-1,57 ; p=0.119)$ (Figura 3A). Não houve variação significativa da diversidade de espécies entre 0 período de estiagem $(X=1,09$; DP $=0,41)$ e chuvoso $(X=1,14 ; D P=0,43)(t=0,62 ; p=0.539)$ (Figura 3B). Também não houve variação significativa do Log da densidade de indivíduos entre o período de estiagem ( $X$ $=3,2 ; \mathrm{DP}=0,59)$ e chuvoso $(\mathrm{X}=3,1 ; \mathrm{DP}=0,57)(\mathrm{t}$ $=-1,45 ; p=0.150$ ) (Figura 3C).

As dez espécies com as maiores densidades relativas totalizam $90 \%$ e $88 \%$ de indivíduos da comunidade da regeneração natural, entre os períodos de estiagem e chuvoso, respectivamente. Contudo, somente quatro espécies foram comuns aos dois períodos, mas com densidades relativas diferentes (Tabela 1).

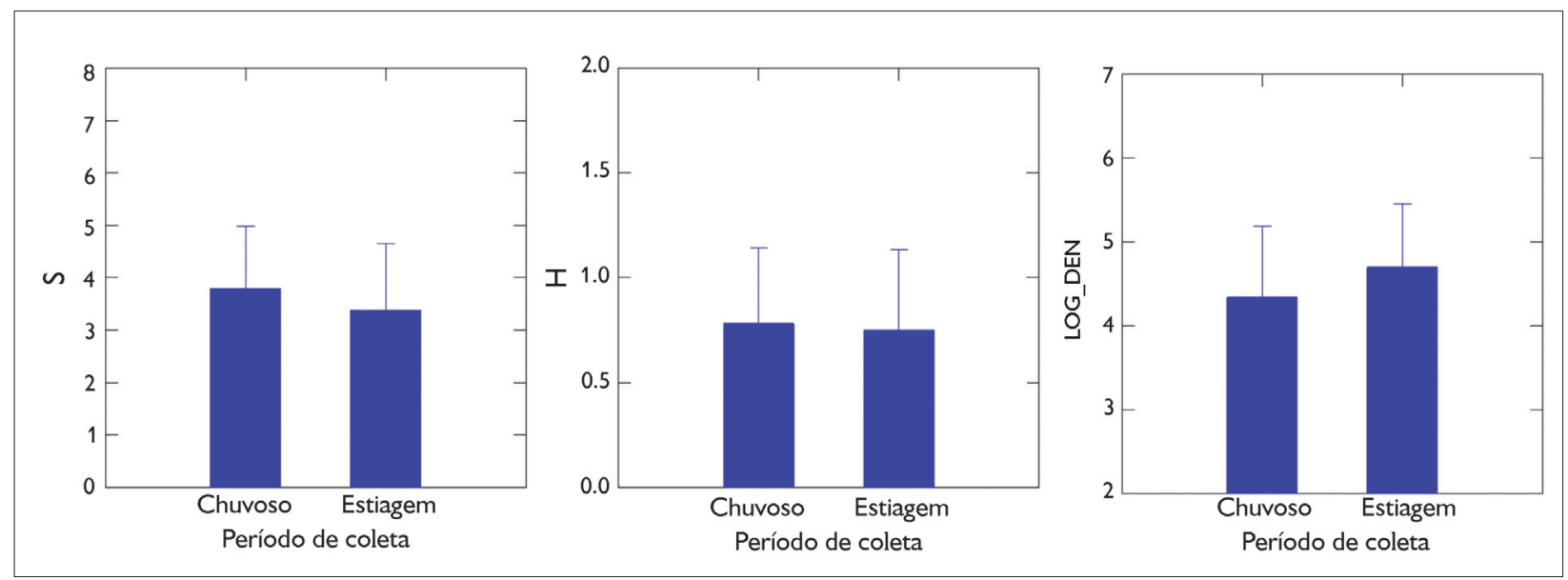

Figura 3. Média e desvio padrão do número de espécies (S), diversidade de espécies (H') e Log da densidade de indivíduos (LON_DEN) da comunidade da regeneração natural dos campos rupestres, entre os períodos chuvoso e de estiagem, na Serra Norte de Carajás, Pará.

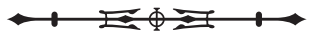


Do total de 67 espécies, 11 (16\%) foram comuns aos períodos de estiagem e chuvoso. Destas, sete espécies tiveram aumento da densidade relativa no período de estiagem em comparação ao chuvoso, variando de 4,3\% (Anthurium lindmanianum Engl.) a 1,320\% (Borreria tenella (Kunth) Cham. \& Schltdl.), e três espécies tiveram uma diminuição da densidade relativa no período de estiagem em relação ao chuvoso, variando de 0,3\% (Sobralia liliastrum Lindl.) a 91,1\% (Cuphea annulata Koehne) (Tabela 2).

\section{LAGOAS SAZONAIS}

Nos lagoas sazonais, foram identificadas 61 espécies, variando de 30 e 41, entre os períodos de estiagem e chuvoso, respectivamente (Apêndice 2). Não houve

Tabela 1. Dez espécies com maior densidade relativa (DR) entre os períodos de estiagem e chuvoso da comunidade da regeneração natural nos campos rupestres da Serra Norte de Carajás, Pará (as espécies comuns a ambos os períodos estão em negrito).

\begin{tabular}{c|c|c|c}
\hline Período de estiagem & & Período chuvoso & \\
\hline Nome científico & DR & Nome científico & DR \\
\hline Axonopus purpusii (Mez) Chase & 54.6 & Axonopus purpusii (Mez) Chase & 28.6 \\
\hline Anthurium lindmanianum Engl. & 9.0 & Dyckia duckei L.B. Sm. & 12.0 \\
\hline Sobralia liliastrum Lindl. & 6.2 & Anthurium lindmanianum Engl. & 9.4 \\
\hline Croton glandulosus L. & 4.9 & Gentianaceae sp. & 8.5 \\
\hline Mimosa acutistipula (Mart.) Benth. & 4.7 & Siphanthera sp. & 7.2 \\
\hline Cuphea antisyphilitica Kunth & 3.8 & Sobralia liliastrum Lindl. & 6.1 \\
\hline Dyckia duckei L.B. Sm. & 2.6 & Cuphea anagalloidea A. St. Hil. & 6.0 \\
\hline Bulbostylis capillaris (L.) Kunth ex C.B. Clarke & 1.7 & Cladonia sp. & 5.0 \\
\hline Vellozia glochidea Pohl & 1.4 & Mesosetum lolifforme (Hochst. ex Steud.) Chase & 2.6 \\
\hline Cuphea annulata Koehne & 1.2 & Paspalum minimum Meyen & 2.5 \\
\hline Total & 90.2 & Total & 88.0 \\
\hline Demais espécies (N $=38)$ & 9.8 & Demais espécies ( $\mathrm{N}=23$ ) & 12.0 \\
\hline
\end{tabular}

Tabela 2. Densidade relativa total e variação da densidade relativa das 11 espécies comuns aos períodos de estiagem e chuvoso da comunidade da regeneração natural dos campos rupestres da Serra Norte de Carajás, Pará.

\begin{tabular}{c|c|c|c}
\hline Nome científico & Período de estiagem (DR) & Período chuvoso (DR) & Variação da DR \\
\hline Anthurium lindmanianum Engl. & 9.0 & 9.4 & 4.3 \\
\hline Axonopus purpusii (Mez) Chase & 54.6 & 28.6 & -47.6 \\
\hline Borreria tenella (Kunth) Cham. \& Schltdl & 0.1 & 0.7 & 1.320 \\
\hline Cassytha filiformis L. & 0.1 & 0.1 & 0.0 \\
\hline Chamaecrista flexuosa (L.) Greene & 0.2 & 0.2 & 37.5 \\
\hline Cladonia sp. & 0.6 & 5.0 & 754.2 \\
\hline Cuphea annulata Koehne & 1.2 & 0.1 & -91.1 \\
\hline Dyckia duckei L.B. Sm. & 2.6 & 12.0 & 361.4 \\
\hline Epidendrum nocturnum Jacq. & 0.4 & 0.5 & 14.0 \\
\hline Sobralia liliastrum Lindl. & 6.2 & 6.1 & -0.3 \\
\hline Turnera breviflora Moura & 0.2 & 0.7 & 200.0 \\
\hline
\end{tabular}

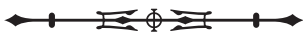


variação significativa da riqueza de espécies entre $\mathrm{O}$ período de estiagem $(X=3,4 ; \mathrm{DP}=1,25)$ e chuvoso $(X$ $=3,8 ; \mathrm{DP}=1,18)(\mathrm{t}=1,85 ; \mathrm{p}=0,07)$ (Figura 4A). Não houve variação significativa da diversidade de espécies entre o período de estiagem $(X=0,75$; $D P=0,38)$ e chuvoso $(X=0,78 ; \mathrm{DP}=0,36)(\mathrm{t}=0,44 ; \mathrm{p}=0.66)$ (Figura 4B). Também não houve variação significativa no
Log da densidade entre o período de estiagem $(X=4,7$; $\mathrm{DP}=0,74)$ e chuvoso $(X=4,3$; $\mathrm{DP}=0,84)(\mathrm{t}=-2,51$; $\mathrm{p}=0,07$ ) (Figura 4C).

As dez espécies com maior densidade relativa totalizam $84 \%$ de indivíduos nas lagoas sazonais entre os períodos de estiagem e chuvoso, e não houve espécies comuns aos dois períodos (Tabela 3).

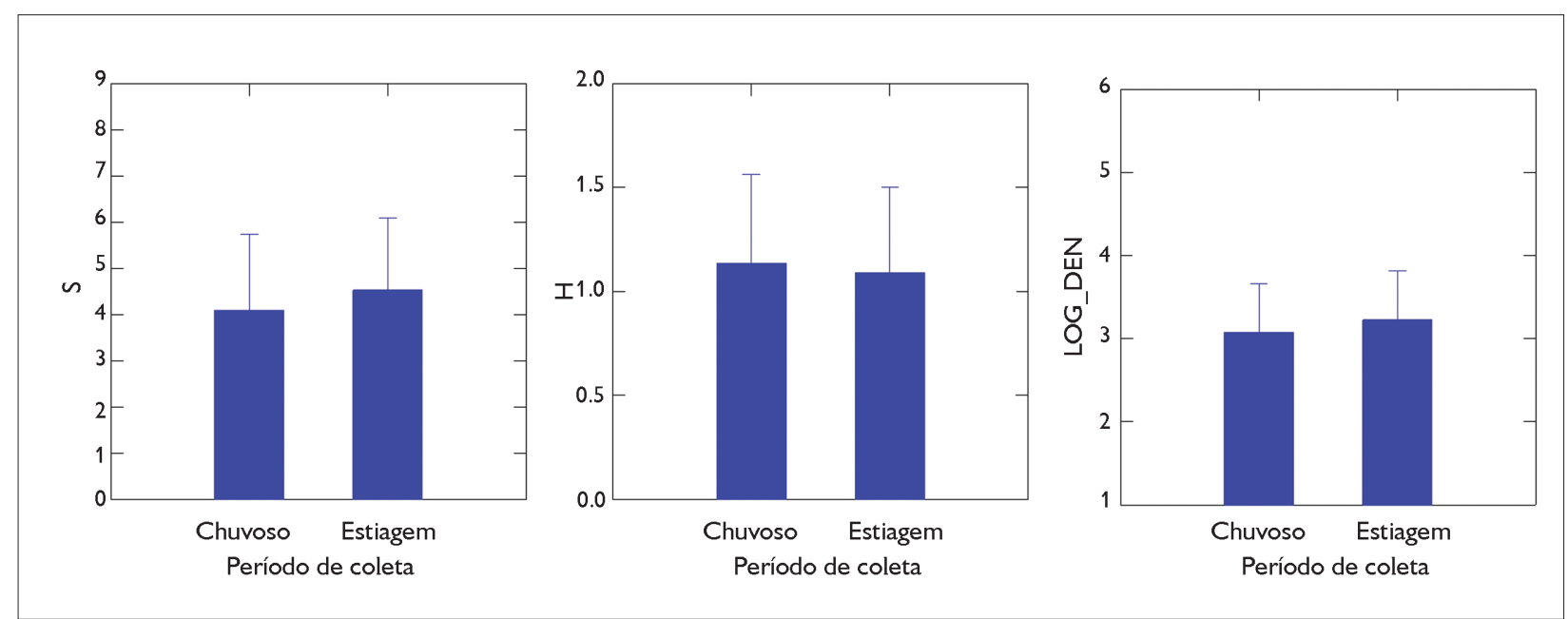

Figura 4. Média e desvio padrão do número de espécies (S), diversidade de espécies (H') e Log da densidade de indivíduos (LON_DEN) da comunidade da regeneração natural nas lagoas sazonais, entre os períodos chuvoso e de estiagem, na Serra Norte de Carajás, Pará.

Tabela 3. Dez espécies com maior densidade relativa (DR) entre os períodos de estiagem e chuvoso da comunidade da regeneração natural das lagoas sazonais nos campos rupestres da Serra Norte de Carajás, Pará.

\begin{tabular}{c|c|c|c}
\hline Período de estiagem & & Período chuvoso & Nome científico \\
\hline Nome científico & DR & DR \\
\hline Rhynchospora cyperoides Mart. & 14.29 & Eleocharis geniculata (L.) Roem. \& Schult. & 26.45 \\
\hline Cyperus laetus Juss. & 14.22 & Mayaca fluviatilis Aubl. & 26.38 \\
\hline Rhynchospora filiformis Vahl & 12.2 & Borreria laevis (Lam.) Griseb. & 5.04 \\
\hline Sida cordifolia L. & 10.67 & Axonopus purpusii (Mez) Chase & 4.92 \\
\hline Fimbristylis cymosa R. Br. & 8.08 & Paspalum pilosum Lam. & 3.95 \\
\hline Eleocharis interstincta (Vahl) Roem. \& Schult. & 6.94 & Panicum parvifolium Lam. & 3.93 \\
\hline Eleocharis geniculata (L.) Roem. \& Schult. & 6.8 & Rhynchospora holoschoenoides (Rich.) Herter & 3.83 \\
\hline Hyptis suaveolens (L.) Poit. & 5.37 & Borreria verticillata (L.) G. Mey. & 3.56 \\
\hline Pontederia tenella Kunth & 2.71 & Rhynchospora graminea Uittien & 3.1 \\
\hline Melochia villosa (Mill.) Fawc. \& Rendle & 2.61 & Hyptis parkeri Benth. & 2.8 \\
\hline Total & 83.89 & Total & 83.96 \\
\hline Demais espécies (N $=$ 20) & 16.11 & Demais espécies (N = 31) & 16.04 \\
\hline
\end{tabular}

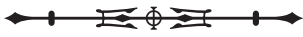


Do total de 61 espécies, dez (16\%) foram comuns aos períodos de estiagem e chuvoso. Destas, quatro espécies tiveram um aumento da densidade relativa no período de estiagem em comparação ao chuvoso, variando de $289 \%$ (Eleocharis geniculata (L.)) a 11,766\% (Borreria verticillata (L.) G. Mey.), e seis espécies tiveram uma diminuição da densidade relativa no período de estiagem em relação ao chuvoso, variando de 33\% (Xyris stenostachya Steyerm.) a 99\% (Nymphoides indica (L.) Kuntze) (Tabela 4).

\section{DISCUSSÃO}

Houve grande diferença na composição da comunidade de plantas da regeneração natural nos campos rupestres e lagoas sazonais nos três enclaves de campos rupestres da serra norte de Carajás, entre o período de estiagem e chuvoso.

A substituição de comunidade de espécies de plantas em relação a fatores abióticos, tais como tipos de solo, altitude, inundação e aspectos topográficos, tem sido descrita em diversos estudos realizados em regiões tropicais (Tuomisto \& Ruokolainen, 1994; Tuomisto, 2003; Ferreira \& Stohlgren, 1999; Ferreira et al., 2010) e temperadas (Itoh et al., 2003; Okuda et al., 2004). Contudo, são inexistentes estudos quantitativos que demonstrem a substituição de comunidade de plantas da regeneração natural em relação à sazonalidade climática nos campos rupestres em substratos ferruginosos.

A distribuição de espécies de plantas em qualquer habitat depende de dois fatores: da capacidade de dispersão dos propágulos (frutos ou sementes) e da disponibilidade de nichos ecológicos favoráveis ao estabelecimento e ao crescimento da regeneração natural (Svenning \& Skov, 2002).

Bastos (1996) não encontrou diferença significativa na riqueza de espécies entre os períodos de estiagem e de chuva nas restingas da costa amazônica paraense, mas informou que existe uma clara mudança da composição de espécies em relação à sazonalidade da precipitação, resultado similar ao encontrados no presente estudo.

Silva (1991), trabalhando nos campos rupestres em substratos ferruginosos da Serra dos Carajás, no estado do Pará, relata que a comunidade de espécies da regeneração natural parece ser adaptada a condições ambientais, a exemplo de baixa fertilidade do solo, baixa capacidade de retenção de água, alta incidência de radiação solar e alta sazonalidade da precipitação ao longo do ano. Contudo, a autora não realizou nenhum estudo quantitativo para corroborar sua hipótese.

Vincent \& Meguro (2008) relatam que, nos campos rupestres sobre afloramento de minério de ferro

Tabela 4. Densidade relativa e variação da densidade relativa das dez espécies comuns aos períodos de estiagem e chuvoso da comunidade da regeneração natural nas lagoas sazonais da Serra Norte de Carajás, Pará.

\begin{tabular}{c|c|c|c}
\hline Nome científico & Período de estiagem (DR) & Período chuvoso (DR) & \% de diferença \\
\hline Aeschynomene sensitiva var. sensitiva & 0.18 & 0.12 & -33 \\
\hline Borreria verticillata (L.) G. Mey. & 0.03 & 3.56 & 11.766 \\
\hline Eleocharis geniculata (L.) & 6.80 & 26.45 & 289 \\
\hline Nymphoides indica (L.) Kuntze & 2.04 & 0.01 & -99 \\
\hline Panicum parvifolium Lam. & 0.08 & 3.93 & 4.812 \\
\hline Paspalum pilosum Lam. & 0.81 & 3.95 & 387 \\
\hline Scleria microcarpa Ness ex Kunth & 1.89 & 0.47 & -75 \\
\hline Sida cordifolia L. & 10.67 & 0.36 & -96 \\
\hline Tonina fluviatilis Aubl. & 0.03 & 1.98 & 6.500 \\
\hline Xyris stenostachya Steyerm. & 0.35 & 0.30 & -14
\end{tabular}

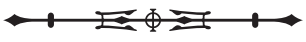


do Quadrilátero Ferrífero em Minas Gerais, as espécies da regeneração natural estão adaptadas às condições abióticas, sendo divididas em grupos que suportam condições de alta insolação, temperaturas elevadas e escassez de água, típicas dos afloramentos rochosos, e as espécies que ocorrem diretamente sobre o substrato endurecido. Outro grupo de espécies é formado por exemplares efêmeros ou geófitas típicas de ambientes sazonalmente inundados e/ou que se estabelecem sobre um substrato mais profundo, ambientes típicos de lagoas sazonais encontrados neste estudo nos campos rupestres.

Esses resultados são importantes, pois, apesar de os campos rupestres e as lagoas sazonais que ocorrem neste estudo e nas restingas litorâneas analisadas por Bastos (1996) serem vegetações bastante distintas em relação à riqueza e à composição de espécies, as vegetações são submetidas a algumas condições abióticas semelhantes, tais como solos extremamente pobres em nutrientes, baixa capacidade de retenção hídrica, alto nível de radiação solar e grande variação na distribuição da precipitação ao longo do ano.

Isto é demonstrado em ecologia e denominado de convergência evolutiva, onde habitats com condições abióticas equivalentes têm espécies que ocupam nichos equivalentes, apesar de as composições de espécies serem totalmente distintas em nível de comunidades e populações.

A dominância de poucas espécies em relação ao total da comunidade da regeneração natural nos campos rupestres e nas lagoas sazonais neste estudo também foi demonstrada por Bastos (1996) para a comunidade de plantas herbáceas das restingas costeiras do estado do Pará.

A substituição de espécies nos campos rupestres e lagoas sazonais neste estudo também foi relatada por Bastos (1996) e Silva (1991), que registraram a existência de dois grupos de comunidade de espécies: o primeiro é representado por espécies que restringem seu período de vida vegetativo e reprodutivo a um período climático (estiagem ou chuvoso) e o segundo, representado por poucas espécies que ocorrem em ambos os períodos climáticos, mas com densidades relativas bem distintas.

Bastos (1996) relata o mesmo padrão para as restingas costeiras paraenses, demonstrando a substituição das comunidades de plantas herbáceas em relação à sazonalidade de precipitação, e uma grande variação na densidade relativa das espécies que ocorrem em ambos os períodos climáticos.

De fato, mais de $90 \%$ do total da precipitação anual nas Serras de Carajás ocorrem no período chuvoso. Desta forma, espera-se encontrar uma flora bem adaptada a essas condições e outro grupo bem adaptado para sobreviver às condições adversas do período de estiagem, no qual as precipitações médias são drasticamente reduzidas.

Estes resultados sugerem que a vegetação da regeneração natural dos campos rupestres sobre afloramento ferrugíneo se distribui ao longo de um gradiente ambiental, determinado tanto pelo substrato, com espécies que se estabelecem diretamente sobre substrato ferruginoso endurecido, como por espécies que colonizam locais com acúmulo de sedimentos e respondem à variação da precipitação entre os períodos de estiagem e chuvoso (Takahasi \& Meirelles, 2014).

Os resultados do presente estudo demonstram que as estratégias de conservação da comunidade de plantas da regeneração natural em campos rupestres e lagoas sazonais analisados, bem como outros tipos de vegetações sujeitas à sazonalidade da precipitação ao longo do ano na Amazônia, não devem ser limitadas a coletas pontuais, mas sim distribuídas ao longo do tempo.

Essa estratégia será a única verdadeiramente eficiente para determinar o conjunto total de espécies da comunidade, sendo o parâmetro mais fundamental para traçar estratégias para a conservação da biodiversidade, especialmente nas comunidades das espécies dos campos rupestres da Serra dos Carajás, no estado do Pará, que se encontram ameaçados pela exploração mineral. 


\section{REFERÊNCIAS}

AB'SABER, A., 1986. Geomorfologia da região. In: J. M. G. ALMEIDA JR. (Org.): Carajás: desafio político, ecologia e desenvolvimento: 88-124. CNPq/Brasiliense, São Paulo.

BASTOS, M. N. C., 1996. Caracterização das formações vegetais da restinga da Princesa, ilha de Algodoal, Pará: 1-290. Tese (Doutorado em Ciências Biológicas - Ecologia de Ecossistemas Costeiros) - Universidade Federal do Pará, Belém.

FERREIRA, L. V. \& T. J. STOHLGREN, 1999. Effects of river level fluctuation on plant species richness, diversity, and distribution in a floodplain forest in Central Amazonia. Oecologia 120(4): 582-587.

FERREIRA, L. V., S. S. ALMEIDA \& P. PAROLIN, 2010. Amazonian white- and blackwater floodplain forests in Brazil: large differences on a small scale. Ecotropica 16(1): 31-41.

INSTITUTO BRASILEIRO DO MEIO AMBIENTE E DOS RECURSOS NATURAIS RENOVÁVEIS (IBAMA), 2003. Plano de manejo para uso múltiplo da Floresta Nacional de Carajás. IBAMA, Brasília.

ITOH, A., T. YAMAKURA, T. OHKUBO, M. KANZAKI, P. A. PALMIOTTO, J. V. LAFRANKIE, P. S. ASHTON \& H. S. LEE, 2003. Importance of topography and soil texture in the spatial distribution of two sympatric dipterocarp trees in a Bornean rainforest. Ecological Research 18(3): 307-320.

OKUDA, T., A. ADACHI, M. SUZUKI, N. A. HUSSEIN, N. MANOKARAN, L. G. SAW, A. H. M. SHARIFF \& P. S. ASHTON, 2004. Local variation of canopy structure in relation to soils and topography and implications for species diversity in a rain forest of peninsular Malaysia. In: E. C. LOSOS \& E. G. LEIGH JR. (Ed.): Tropical forest diversity and dynamism: finding from a large-scale pilot network: 221-240. The University of Chicago Press, Chicago and London.

RAYOL, B. P., 2006. Análise florística e estrutural da vegetação xerofítica das savanas metalófilas na Floresta Nacional de Carajás: subsídios à conservação: 1-76. Dissertação (Mestrado em Botânica) - Universidade Federal Rural da Amazônia/Museu Paraense Emílio Goeldi, Belém.

SCHAEFER, C. E. G. R., F. N. B. SIMAS, B. A. F. MENDONÇA, A. S. S. RIBEIRO, G. R. CORREA \& W. F. JÚNIOR, 2008. Geodiversidade dos ambientes de canga na região de Carajás - Pará: 1-75. Relatório técnico. Vale do Rio Doce, Belém.

SECCO, R. \& A. L. MESQUITA, 1983. Notas sobre a vegetação de canga da Serra Norte - I. Boletim do Museu Paraense Emílio Goeldi, Nova Série Botânica 59: 1-13.

SECCO, R. S. \& M. G. A. LOBO, 1988. Considerações taxonômicas e ecológicas sobre a flora dos "campos rupestres" da Serra dos Carajás. Boletim da FBCN 3(23): 30-44.
SILVA, M. F. F., 1989. Aspectos ecológicos da vegetação que cresce sobre canga hematítica em Carajás - PA. Tese (Doutorado em Botânica) - Instituto Nacional de Pesquisas da Amazônia, Belém.

SILVA, M. F. F., 1991. Análise florística da vegetação que cresce sobre canga hematítica em Carajás - Pará (Brasil). Boletim do Museu Paraense Emílio Goeldi, série Botânica 7(1): 79-105.

SILVA, M. F. F. \& N. A. ROSA, 1990. Estudos botânicos na área do projeto-ferro Carajás/Serra Norte. I. Aspectos fito-ecológicos dos campos rupestres. Anais do Congresso Nacional de Botânica 35: 367-379.

SILVA, M. F. F., R. S. SECCO \& M. G. LOBO, 1996. Aspectos ecológicos da vegetação rupestre da Serra dos Carajás, estado do Pará, Brasil. Acta Amazonica 26(1-2): 17-44.

SVENNING, J.-C. \& F. SKOV, 2002. Mesoscale distribution of understorey plants in temperate forest (Kolo, Dennmark): the importance of environment and dispersal. Plant Ecology 160: 169-185.

TAKAHASI, A. \& S. T. MEIRELLES, 2014. Ecologia da vegetação herbácea de bancadas lateríticas (cangas) em Corumbá, MS, Brasil. Hoehnea 41(4): 515-528.

THE ANGIOSPERM PHYLOGENY GROUP, 2009. An update of the Angiosperm Phylogeny Group classification for the orders and families of flowering plants: APG III. Botanical Journal of the Linnean Society 161(2): 105-121.

TUOMISTO, H., 2003. Floristic patterns along a 43-km long transect in Amazonian rain forest. Journal of Ecology 91(5): 743-756.

TUOMISTO, H. \& K. RUOKOLAINEN, 1994. Distribution of Pteridophyta an Melastomataceae along an edaphic gradient in an Amazonian rain forest. Journal of Vegetation Science 5(1): 25-34.

VASCONCELOS, M. F., 2011. O que são campos rupestres e campos de altitude nos topos de montanhas do leste do Brasil? Revista Brasileira de Botânica 34(2): 241-246.

VELOSO, H. P., A. L. RANGEL FILHO \& J. C. A. LIMA, 1991. Classificação da vegetação brasileira, adaptada a um sistema universal. IBGE, Rio de Janeiro.

VINCENT, R. C. \& M. MEGURO, 2008. Influence of soil properties on the abundance of plant species in ferruginous rocky soils vegetation, southeastern Brazil. Brazilian Journal of Botany 31(3): 377-388.

ZAR, J. H., 2010. Biostastical analysis: 5.ed. Prentice-Hall, Englewwod Cliffs, New Jersey. 
Apêndice 1. Densidade relativa das espécies da comunidade de plantas herbáceas nos campos rupestres da Serra Norte de Carajás, Pará, em relação aos períodos de estiagem e chuvoso.

(Continua)

\begin{tabular}{|c|c|c|c|c|}
\hline $\mathrm{N}$ & Nome científico & Família & Período de estiagem & Período chuvoso \\
\hline 1 & Aechmea sp. & Bromeliaceae & & 0.11 \\
\hline 2 & Aeschynomene sensitiva var. sensitiva & Fabaceae & 0.22 & \\
\hline 3 & Alibertia myrciifolia K. Schum. & Rubiaceae & 0.32 & \\
\hline 4 & Anemopaegma scabriusculum Mart. ex DC. & Bignoniaceae & 0.16 & \\
\hline 5 & Anthurium lindmanianum Engl. & Araceae & 9.03 & 9.42 \\
\hline 6 & Axonopus purpusii (Mez) Chase & Poaceae & 54.59 & 28.6 \\
\hline 7 & Banisteriopsis malifolia var. appressa B. Gates & Malpighiaceae & 0.43 & \\
\hline 8 & Bauhinia pulchella Benth. & Fabaceae & 0.43 & \\
\hline 9 & Bidens bipinnata $\mathrm{L}$. & Asteraceae & & 1.15 \\
\hline 10 & Borreria semiamplexicaule E.L. Cabral & Rubiaceae & & 0.93 \\
\hline 11 & Borreria tenella (Kunth) Cham. \& Schltdl. & Rubiaceae & 0.05 & 0.71 \\
\hline 12 & Borreria tenuis DC. & Rubiaceae & & 2.36 \\
\hline 13 & Bulbostylis capillaris (L.) Kunth ex C.B. Clarke & Cyperaceae & 1.73 & \\
\hline 14 & Byrsonima eugeniifolia Sandwith & Malpighiaceae & 0.16 & \\
\hline 15 & Callisthene microphylla Warm. & Vochysiaceae & 0.05 & \\
\hline 16 & Cassytha filiformis L. & Lauraceae & 0.05 & 0.05 \\
\hline 17 & Catasetum sp. & Orchidaceae & & 0.55 \\
\hline 18 & Cereus sp. & Cactaceae & 0.11 & \\
\hline 19 & Chamaecrista flexuosa (L.) Greene & Fabaceae & 0.16 & 0.22 \\
\hline 20 & Cissus apendiculata Lombardi & Vitaceae & 0.05 & \\
\hline 21 & Cladonia sp. & Cladoniaceae & 0.59 & 5.04 \\
\hline 22 & Crotalaria maypurensis Kunth & Fabaceae & & 0.27 \\
\hline 23 & Croton glandulatus Vell. & Euphorbiaceae & 4.92 & \\
\hline 24 & Croton tomentosus (Lour.) Müll. Arg. & Euphorbiaceae & 0.27 & \\
\hline 25 & Cuphea anagalloidea A. St.-Hil. & Lythraceae & & 6.03 \\
\hline 26 & Cuphea annulata Koehne & Lythraceae & 1.24 & 0.11 \\
\hline 27 & Cuphea antisyphilitica Kunth & Lythraceae & 3.84 & \\
\hline 28 & Desmodium canum Schinz \& Thell. & Fabaceae & 0.16 & \\
\hline 29 & Dioclea virgata var. crenata R.H. Maxwell & Fabaceae & 0.92 & \\
\hline 30 & Dioclea virgata (Rich.) Amshoff & Fabaceae & 0.22 & \\
\hline 31 & Dyckia duckei L.B. Sm. & Bromeliaceae & 2.59 & 11.95 \\
\hline 32 & Epidendrum nocturnum Jacq. & Orchidaceae & 0.43 & 0.49 \\
\hline 33 & Erythroxylum ligustrinum var. carajasense Plowman & Erythroxylaceae & 0.22 & \\
\hline 34 & Erythroxylum nelson-rosae Plowman & Erythroxylaceae & 0.32 & \\
\hline 35 & Habenaria sp. & Orchidaceae & & 0.05 \\
\hline 36 & Ipomoea carajasensis D.F. Austin & Convolvulaceae & 0.38 & \\
\hline
\end{tabular}


Apêndice 1.

\begin{tabular}{|c|c|c|c|c|}
\hline $\mathrm{N}$ & Nome científico & Família & Período de estiagem & Períndo chuvoso \\
\hline 37 & Lippia grandis Schau & Verbenaceae & 0.11 & \\
\hline 38 & Mandevilla hirsuta (Rich.) K. Schum. & Apocynaceae & 0.05 & \\
\hline 39 & Mandevilla angustifolia (Malme) Woodson & Apocynaceae & 0.22 & \\
\hline 40 & Memora allamandiflora Bureau ex K. Schum. & Bignoniaceae & 0.49 & \\
\hline 41 & Mesosetum loliforme (Hochst. ex Steud.) Chase & Poaceae & & 2.58 \\
\hline 42 & Mimosa acutistipula (Mart.) Benth. & Fabaceae & 4.65 & \\
\hline 43 & Mimosa pigra L. & Fabaceae & & 0.49 \\
\hline 44 & Morfotipo 1 & Gentianaceae & & 8.49 \\
\hline 45 & Norantea guianensis Aubl. & Marcgraviaceae & 0.38 & \\
\hline 46 & Panicum nervosum Lam. & Poaceae & 0.27 & \\
\hline 47 & Panicum parvifolium Lam. & Poaceae & & 0.22 \\
\hline 48 & Paspalum carinatum Humb. \& Bonpl. ex Flüggé & Poaceae & & 0.44 \\
\hline 49 & Paspalum minimum Meyen & Poaceae & & 2.52 \\
\hline 50 & $\begin{array}{c}\text { Peperomia macrostachyos var. nematostachya (Link) } \\
\text { Trel. \& Yunck }\end{array}$ & Piperaceae & 0.43 & \\
\hline 51 & Periandra mediterranea (Vell.) Taub. & Fabaceae & 0.86 & \\
\hline 52 & Philodendron acutatum Schott & Araceae & 0.16 & \\
\hline 53 & Pilocarpus microphyllus Stapf ex Wardleworth & Rutaceae & 0.16 & \\
\hline 54 & Psychotria barbiflora DC. & Rubiaceae & & 0.16 \\
\hline 55 & Rhynchospora barbata (Vahl) Kunth & Cyperaceae & & 0.93 \\
\hline 56 & Riencourtia glomerata Cass. & Asteraceae & & 1.04 \\
\hline 57 & Sapium marginatum Müll. Arg. & Euphorbiaceae & 0.05 & \\
\hline 58 & Sauvagesia erecta L. & Ochnaceae & & 0.33 \\
\hline 59 & Siphanthera sp. & Melastomataceae & & 7.23 \\
\hline 60 & Sobralia liliastrum Lindl. & Orchidaceae & 6.16 & 6.14 \\
\hline 61 & Syngonanthus fertilis (Körn.) Ruhland & Eriocaulaceae & & 0.33 \\
\hline 62 & Tillandsia bulbosa Hook. & Bromeliaceae & 0.05 & \\
\hline 63 & Turnera breviflora Moura & Passifloraceae & 0.22 & 0.66 \\
\hline 64 & Turnera glaziovii Urb. & Passifloraceae & & 0.16 \\
\hline 65 & Vellozia glochidea Pohl. & Velloziaceae & 1.41 & \\
\hline 66 & Vernonia muricata DC. & Asteraceae & & 0.22 \\
\hline
\end{tabular}


Apêndice 2. Densidade relativa das espécies da comunidade de plantas herbáceas amostradas nas lagoas sazonais da Serra Norte de Carajás, Pará, em relação aos períodos de estiagem e chuvoso.

(Continua)

\begin{tabular}{|c|c|c|c|c|}
\hline N & Nome científico & Família & Período de estiagem & Período chuvoso \\
\hline 1 & Aeschynomene evenia C. Wright & Fabaceae & & 0.3 \\
\hline 2 & Aeschynomene paniculata Willd. ex Vogel & Fabaceae & 0.47 & \\
\hline 3 & Aeschynomene sensitiva var. sensitiva & Fabaceae & 0.18 & 0.12 \\
\hline 4 & Andropogon leucopogon Nees & Poaceae & & 0.37 \\
\hline 5 & Axonopus purpusii (Mez) Chase & Poaceae & & 4.92 \\
\hline 6 & Borreria laevis (Lam.) Griseb. & Rubiaceae & & 5.04 \\
\hline 7 & Borreria verticillata (L.) G. Mey. & Rubiaceae & 0.03 & 3.56 \\
\hline 8 & Bulbostylis capillaris (L.) Kunth ex C.B. Clarke & Cyperaceae & 2.13 & \\
\hline 9 & Cassytha filiformis $\mathrm{L}$. & Lauraceae & & 0.04 \\
\hline 10 & Cuphea antisyphilitica Kunth. & Lythraceae & & 0.42 \\
\hline 11 & Cyperus globosus Forssk. & Cyperaceae & 0.14 & \\
\hline 12 & Cyperus haspan L. & Cyperaceae & & 2.77 \\
\hline 13 & Cyperus laetus Presl. & Cyperaceae & 14.22 & \\
\hline 14 & Digitaria horizontalis Willd. & Poaceae & 0.72 & \\
\hline 15 & $\begin{array}{c}\text { Echinodorus tenellus (Mart. ex Schult. \& Schult. f.) } \\
\text { Buchenauz }\end{array}$ & Alismataceae & 0.38 & \\
\hline 16 & Eleocharis geniculata (L.) Roem. \& Schult. & Cyperaceae & 6.8 & 26.45 \\
\hline 17 & Eleocharis interstincta (Vahl) Roem. \& Schult. & Cyperaceae & 6.94 & \\
\hline 18 & Fimbristylis cymosa R. Br. & Cyperaceae & 8.08 & \\
\hline 19 & Fimbristylis sp. R. Br. & Cyperaceae & & 0.5 \\
\hline 20 & Hemidiodia sp. Subarb. & Rubiaceae & & 0.09 \\
\hline 21 & Hyptis parkeri Benth. & Lamiaceae & & 2.8 \\
\hline 22 & Hyptis suaveolens (L.) Poit. & Lamiaceae & 5.37 & \\
\hline 23 & Ludwigia hyssopifolia (G. Don) Exell & Onagraceae & & 0.17 \\
\hline 24 & Ludwigia nervosa (Poir.) H. Hara & Onagraceae & & 0.02 \\
\hline 25 & Mayaca fluviatilis Aubl. & Mayacaceae & & 26.38 \\
\hline 26 & Melochia hirsuta Cav. & Malvaceae & & 0.16 \\
\hline 27 & Melochia villosa (Mill.) Fawc. \& Rendle & Malvaceae & 2.61 & \\
\hline 28 & Mesosetum loliiforme (Hochst. ex Steud.) Chase & Poaceae & & 2.48 \\
\hline 29 & Mimosa pigra L. & Fabaceae & & 1.35 \\
\hline 30 & Mimosa pudica L. & Fabaceae & & 0.06 \\
\hline 31 & Mimosa sensitiva $L$. & Fabaceae & & 0.06 \\
\hline 32 & Mimosa skinneri var. carajarum Barneby & Fabaceae & & 0.02 \\
\hline 33 & Mimosa somnians Humb. \& Bonpl. ex Willd. & Fabaceae & & 0.02 \\
\hline 34 & Nymphoides indica (L.) Kuntze & Fabaceae & 2.04 & 0.01 \\
\hline 35 & Oldenlandia corymbosa L. & Rubiaceae & & 0.02 \\
\hline
\end{tabular}


Apêndice 2.

(Conclusão)

\begin{tabular}{|c|c|c|c|c|}
\hline $\mathrm{N}$ & Nome científico & Família & Período de estiagem & Período chuvoso \\
\hline 36 & Paepalanthus sp. Ruhland. & Eriocaulaceae & 1.52 & \\
\hline 37 & Paepalanthus tenuis (Kunth) Kunth & Eriocaulaceae & 0.18 & \\
\hline 38 & Panicum parvifolium Lam. & Poaceae & 0.08 & 3.93 \\
\hline 39 & Paspalum parviflorum Rhode ex Fluggé & Poaceae & & 2.69 \\
\hline 40 & Paspalum pilosum Lam. & Poaceae & 0.81 & 3.95 \\
\hline 41 & Paspalum sp. Lam. & Poaceae & 2.13 & \\
\hline 42 & Paspalum tenuis M.E. Jones & Poaceae & 1.37 & \\
\hline 43 & Phyllanthus niruri L. & Phyllanthaceae & & 0.09 \\
\hline 44 & Polygala adenophora DC. & Polygalaceae & & 0.32 \\
\hline 45 & Pontederia sp. L. & Pontederiaceae & 2.71 & \\
\hline 46 & Pontederia tenuis $\mathrm{L}$. & Pontederiaceae & 0.32 & \\
\hline 47 & Rhynchospora barbata (Vahl) Kunth & Cyperaceae & & 0.07 \\
\hline 48 & Rhynchospora ciliata (G. Mey.) Kük. & Cyperaceae & 1.3 & \\
\hline 49 & Rhynchospora corymbosa (L.) Britton & Cyperaceae & & 0.5 \\
\hline 50 & Rhynchospora cyperoides Mart. & Cyperaceae & 14.29 & \\
\hline 51 & Rhynchospora filiformis Vahl & Cyperaceae & 12.2 & \\
\hline 52 & Rhynchospora graminea Uittien & Cyperaceae & & 3.1 \\
\hline 53 & Rhynchospora holoschoenoides (Rich.) Herter & Cyperaceae & & 3.83 \\
\hline 54 & Rhynchospora sp. Uittien. & Cyperaceae & & 0.19 \\
\hline 55 & Scleria bracteata Cav. & Cyperaceae & 0.03 & \\
\hline 56 & Scleria microcarpa Nees ex Kunth & Cyperaceae & 1.89 & 0.47 \\
\hline 57 & Sida cordifolia L. & Malvaceae & 10.67 & 0.36 \\
\hline 58 & Stylosanthes angustifolia Vogel & Fabaceae & & 0.02 \\
\hline 59 & Tonina fluviatilis Aubl. & Eriocaulaceae & 0.03 & 1.98 \\
\hline 60 & Turnera breviflora Moura & Passifloraceae & & 0.06 \\
\hline 61 & Xyris stenostachya Steyerm. & Xyridaceae & 0.35 & 0.3 \\
\hline
\end{tabular}

